\title{
Numerical Simulation of Bubble Growth in Liquid Jet Impingement on a Hot Plate*
}

\author{
Kyungmin $\mathrm{KIM}^{* *}$ and Gihun SON** \\ ** Department of Mechanical Engineering, Sogang University \\ Seoul 121-742, South Korea \\ E-mail: gihun@sogang.ac.kr
}

\begin{abstract}
Complete numerical simulations are performed for bubble growth in water jet impingement on a hot plate. The governing equations for the conservation of mass, momentum and energy are numerically solved in the liquid, vapor and air phases. The liquidvapor and liquid-air interfaces are tracked by a level-set method which is modified to include the effect of phase change at the liquid-vapor interface. The level-set approach is combined with a non-equilibrium $\mathrm{k}-\varepsilon$ turbulence model. The effects of jet velocity, jet temperature and wall superheat on the bubble growth in water jet and the associated flow and heat transfer are quantified.
\end{abstract}

Key words : Bubble Growth, Jet Impingement, $k-\varepsilon$ Turbulence Model, Level-Set Method

\section{Introduction}

Boiling in free-surface liquid jet impingement is one of the most efficient modes of heat transfer. It has been utilized as the primary cooling mechanism in a steel plate manufacturing process and also receives increasing attention as an effective cooling method for high-powered electronic chips. The jet impingement boiling has been extensively investigated in the literature, including Wolf et al. ${ }^{(1)}$, Hall et al. ${ }^{(2)}$ and Karwa et al. ${ }^{(3)}$. However, the boiling phenomena in free-surface liquid jet impingement are still not well understood because of the complexity of the liquid-air and liquid-vapor interfaces that evolve, merge and break up in time, and the flow and temperature fields influenced by the interfacial motion.

Recently, numerical simulations have been performed to clarify and predict the characteristics of flow and heat transfer in free-surface jet impingement. Fujimoto et al. ${ }^{(4)}$ investigated the convective heat transfer of a circular impinging water jet on a hot solid by solving the Navier-Stokes equation for incompressible laminar flow. The free surface or liquid-gas interface was tracked by using a marker-and-cell (MAC) method, in which the interface is represented explicitly by the line segments and the end points of each segment are moved in the Lagrangian manner. The solid temperature was assumed to be maintained below the boiling temperature. Their predicted flow structures and local Nusselt numbers were comparable to the experimental data. A more detailed analysis of free-surface jet was made by Tong ${ }^{(5)} \mathrm{em}^{\mathrm{e}}$ ploying the volume-of-fluid (VOF) method, in which interface is tracked by the liquid volume fraction. The effects of several key parameters, such as the jet-inlet velocity profile, Reynolds number and jet elevation, on the flow and heat transfer were investigated. However, their analysis did not include the effect of turbulence. Gradeck et al. ${ }^{(6)}$ studied experimentally and numerically a free-surface jet on a moving plate. They employed the VOF method and a nonlinear $k-\varepsilon$ turbulence model to investigate the effects of jet velocity, plate velocity and nozzle geometry on the impinging jet. Son et al. ${ }^{(7)}$ performed numerical simulation of free-surface jet impingement using a level-set method, in which the interface is tracked by a smooth distance function. The level-set approach is combined with a non-equilibrium $k$ - $\varepsilon$ turbulence model to investigate the flow and cooling characteristics of impinging jets. The prediction of interface shape and heat transfer coefficient for a circular impinging water jet showed a good agreement

*Received 23 Jan., 2012 (No. 12-0032) [DOI: 10.1299/jtst.7.237]

Copyright (C) 2012 by JSME 
with the experimental data available in the literature.

Few computations have been made for boiling in free-surface jet impingement. While developing a boundary-layer model for the jet impingement boiling, Mikielewicz et al. ${ }^{\left({ }^{()}\right.}$introduced a blowing velocity to accounts for the transverse transport of momentum caused by bubble growth. The blowing velocity was assumed to be constant and approximated from the evaporation heat flux without considering the bubble motion. Omar et al. ${ }^{(9)}$ also presented a boundary-layer formulation including the additional diffusivities due to the bubble-induced mixing. The diffusivities were correlated with the jet parameters and surface temperature from their experimental data. Using the commercial CFD code FLUENT, Naramuchi et al. ${ }^{(10)}$ computed the boiling phenomena in impinging jets for power electronics cooling. The predicted boiling curves for submerged jets were comparable to the experimental data. However, their analysis based on the Eulerian multiphase model requires a number of empirical correlations for evaluating the exchange of mass, momentum and energy between the different phases.

Complete numerical simulations for bubble growth and boiling heat transfer without including empirical correlations for the interfacial characteristics were also reported in the literature. Efforts were made for computation of pool boiling on a solid wall using moving-grid $\operatorname{methods}^{(11)(12)}$, a front-tracking $\operatorname{method}^{(13)}$, the volume-of-fluid method ${ }^{(14)}$, and a level-set $\operatorname{method}^{(15)(16)}$. However, the complete numerical simulation was not extended to the boiling case in free-surface jet impingement.

In this study, we develop a numerical method for simulation of bubble growth in freesurface liquid jet impingement. The liquid-vapor and liquid-air interfaces are tracked by a level-set method which is modified to include the effect of phase change at the liquid-vapor interface. The level-set approach is combined with the non-equilibrium $k$ - $\varepsilon$ model of Shyy et al. ${ }^{(17)}$. The method is applied to investigate the effects of jet velocity, jet temperature and wall superheat on the bubble growth and boiling heat transfer in water jet.

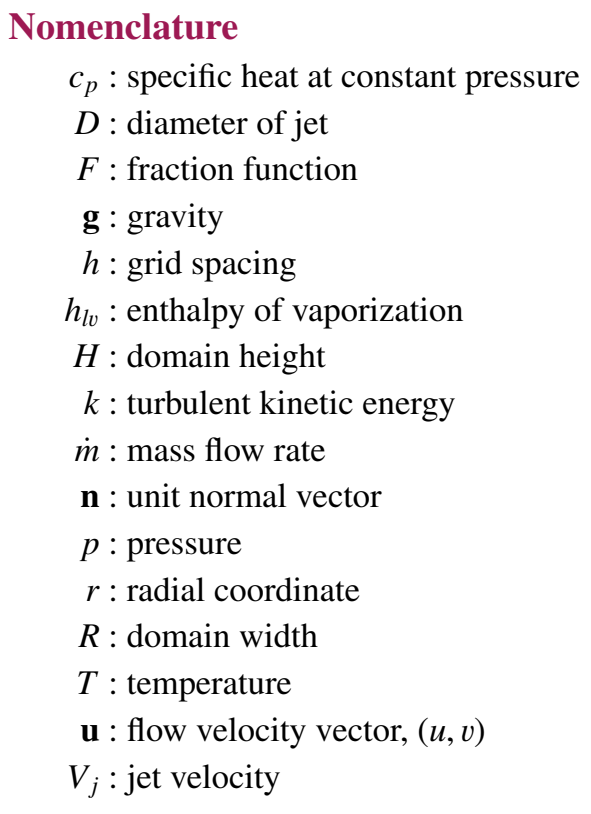

Greek symbols

$$
\begin{aligned}
& \alpha: \text { discontinuous step function } \\
& \varepsilon: \text { turbulent dissipation rate } \\
& \kappa: \text { interface curvature } \\
& \lambda: \text { thermal conductivity } \\
& \tau_{w}: \text { waiting time } \\
& \mu: \text { viscosity } \\
& v_{t}: \text { turbulent kinematic viscosity } \\
& \rho: \text { density } \\
& \sigma: \text { surface tension coefficient } \\
& \phi: \text { level-set function }
\end{aligned}
$$

$$
\begin{aligned}
& \text { subscripts } \\
a, v & : \text { air, vapor } \\
g, l & : \text { gas, liquid } \\
j & : \text { jet } \\
s u b & : \text { subcooling } \\
\text { sat } & : \text { saturation } \\
w & : \text { wall }
\end{aligned}
$$

\section{Numerical analysis}

Figure 1 shows the configuration used for simulation of bubble growth in liquid jet impingement. We assume that the flows are axisymmetric, all fluid properties are constant in each phase, and the vapor phase is at the saturation temperature. The contact angle at the bubble-wall contact line is taken to be $90^{\circ}$ and the evaporation heat flux from a liquid microlayer, which forms near the contact line, is not included in the present analysis. The interface 


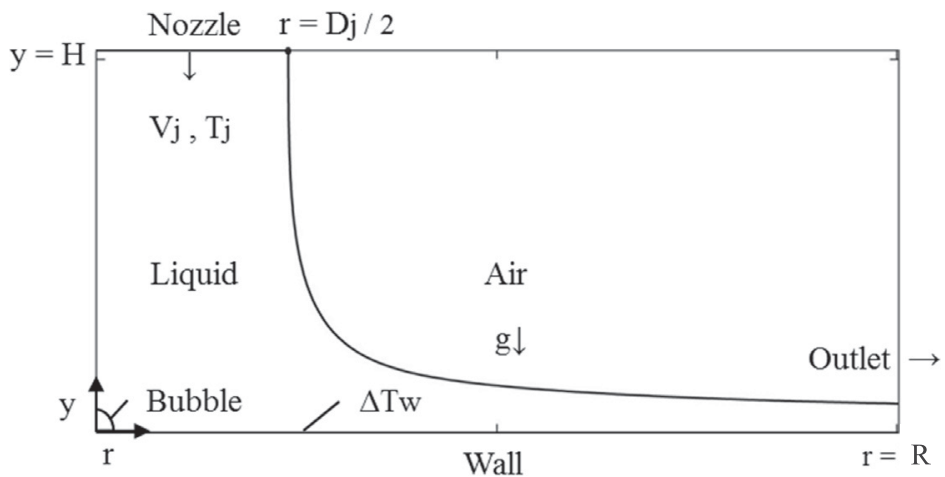

Fig. 1 Computational domain and boundary conditions

separating the liquid-air or liquid-vapor phases is tracked by the level-set (LS) function, which is defined as a signed distance from the interface. The negative sign is chosen for the gas (air or vapor) phase and the positive sign for the liquid phase. To distinguish the air and vapor regions, we introduce a step function $\alpha_{a}$, which is defined as $\alpha_{a}=1$ for the air region and $\alpha_{a}=0$ for the vapor region.

Combining the numerical approaches used by Son et al. ${ }^{(7)}$ and Son and $\operatorname{Dhir}^{(16)}$, the governing equations for conservation of mass, momentum, energy, turbulent energy and turbulent dissipation rate for liquid-gas region can be written as

$$
\begin{aligned}
\nabla \cdot \mathbf{u} & =v_{l v} \dot{m} \mathbf{n} \cdot \nabla \alpha_{l} \\
\rho \frac{D \mathbf{u}}{D t} & =-\nabla p+\rho \mathbf{g}-\sigma \kappa \nabla \alpha_{l}+\nabla \cdot\left(\mu+\rho v_{t}\right)\left[\nabla \mathbf{u}+(\nabla \mathbf{u})^{T}\right] \\
\rho c_{p} \frac{D T}{D t} & =\nabla \cdot\left(\lambda+\rho c_{p} \frac{v_{t}}{P r_{T}}\right) \nabla T \quad \text { if } \phi>0 \text { or } \alpha_{a}=1 \\
T & =T_{s a t} \quad \text { if } \phi \leq 0 \text { and } \alpha_{a}=0 \\
\rho \frac{D k}{D t} & =\nabla \cdot\left(\mu+\rho \frac{v_{t}}{P r_{k}}\right) \nabla k+\rho G-\rho \varepsilon \\
\rho \frac{D \varepsilon}{D t} & =\nabla \cdot\left(\mu+\rho \frac{v_{t}}{P r_{\varepsilon}}\right) \nabla \varepsilon+c_{1} \rho \frac{\varepsilon}{k} G-c_{2} \rho \frac{\varepsilon^{2}}{k}
\end{aligned}
$$

where

$$
\begin{aligned}
\alpha_{l} & =1 \quad \text { if } \quad \phi>0 \\
& =0 \quad \text { if } \quad \phi \leq 0 \\
\dot{m} & =\mathbf{n} \cdot \lambda \nabla T / h_{l v} \\
\kappa & =\nabla \cdot\left(\frac{\nabla \phi}{|\nabla \phi|}\right) \\
\rho & =\rho_{g}(1-F)+\rho_{l} F \\
\mu^{-1} & =\mu_{g}^{-1}(1-F)+\mu_{l}^{-1} F \\
\lambda^{-1} & =\lambda_{g}^{-1}(1-F)+\lambda_{l}^{-1} F \\
v_{t} & =c_{\mu} \frac{k^{2}}{\varepsilon} \exp \left[\frac{-3.4}{\left(1+0.02 \rho k^{2} / \varepsilon \mu\right)^{2}}\right] \\
G & =v_{t}\left[\nabla \mathbf{u}+(\nabla \mathbf{u})^{T}\right]:(\nabla \mathbf{u}) \\
c_{1} & =1.15+0.25 \frac{G}{\varepsilon} ; \quad c_{2}=1.45+0.45 \frac{G}{\varepsilon} \\
c_{\mu} & =0.09 ; \quad \operatorname{Pr}_{T}=0.9 ; \quad \operatorname{Pr}_{k}=0.89 ; \quad \operatorname{Pr}_{\varepsilon}=1.15
\end{aligned}
$$

Here, $\alpha_{\phi}$ is the discontinuous step function rather than the smoothed step function varying over 
several grid spacings and the interface curvature $\kappa$ is evaluated by the smooth LS function. The turbulent viscosity $v_{t}$ is formulated to include the effects of non-equilibrium and low Reynolds number. The effective density $\rho$ and viscosity $\mu$ are evaluated from a fraction function $F$, which is described by Son and Dhir ${ }^{(16)}$.

In the LS formulation, the interface is described as $\phi=0$. The zero level set of $\phi$ is advanced as

$$
\frac{\partial \phi}{\partial t}=-\mathbf{U} \cdot \nabla \phi
$$

where the interface velocity $\mathbf{U}$ is obtained as

$$
\mathbf{U}=\mathbf{u}+\frac{\dot{m}}{\rho} \mathbf{n}
$$

The LS function is reinitialized to a distance function from the interface by obtaining a steadystate solution of the equation

$$
\frac{\partial \phi}{\partial \tau}=S(\phi)(1-|\nabla \phi|)
$$

where

$$
\begin{aligned}
S(\phi) & =0 & & \text { if } \quad|\phi| \leq h / 2 \\
& =\frac{\phi}{\sqrt{\phi^{2}+h^{2}}} & & \text { otherwise }
\end{aligned}
$$

Here $h$ is a grid spacing and $\tau$ is an iteration step. The formulation given by Eq. (19) implies that a near-zero level set rather than $\phi=0$ is used as the immobile boundary condition during the reinitialization procedure ${ }^{(18)}$. The step function $\alpha_{a}$ is initialized for the air region and tracked by the LS function $\phi$ as described by Suh and Son ${ }^{(18)}$.

The governing equations are spatially discretized on a staggered grid system in which the velocity components are defined at cell faces whereas the other dependent variables at cell centers. A second-order ENO scheme is used for the convection terms and the distance function, and second-order central difference scheme for the other terms. While discretizing the governing equations temporally, the convection and source terms are treated by a firstorder explicit scheme and the diffusion terms by a fully implicit scheme.

The boundary conditions used in this study are as follows (refer to Figure 1). At the jet inlet $\left(0 \leq r \leq D_{j} / 2, y=H\right)$

$$
u=0 \quad v=V_{j} \quad T=T_{j} \quad \frac{\partial \phi}{\partial y}=0
$$

At the top plane except the jet inlet $\left(D_{j} / 2 \leq r \leq R, y=H\right)$

$$
v=0 \quad \frac{\partial u}{\partial y}=\frac{\partial T}{\partial y}=\frac{\partial \phi}{\partial y}=0
$$

At the bottom wall $(y=0)$

$$
u=v=0 \quad T=T_{w} \quad \frac{\partial \phi}{\partial y}=0
$$

At the axi-symmetric plane $(r=0)$

$$
u=0 \quad \frac{\partial v}{\partial r}=\frac{\partial T}{\partial r}=\frac{\partial \phi}{\partial r}=0
$$

At the outlet $(r=R)$

$$
p=0 \quad \frac{\partial u}{\partial r}=\frac{\partial T}{\partial r}=\frac{\partial \phi}{\partial r}=0
$$

As the initial conditions, we use

$$
\phi=-r+D_{j} / 2 \quad u=0 \quad T=T_{j} \quad v=0 \text { if } \phi<0 ; \quad v=V_{j} \text { if } \phi \geq 0
$$



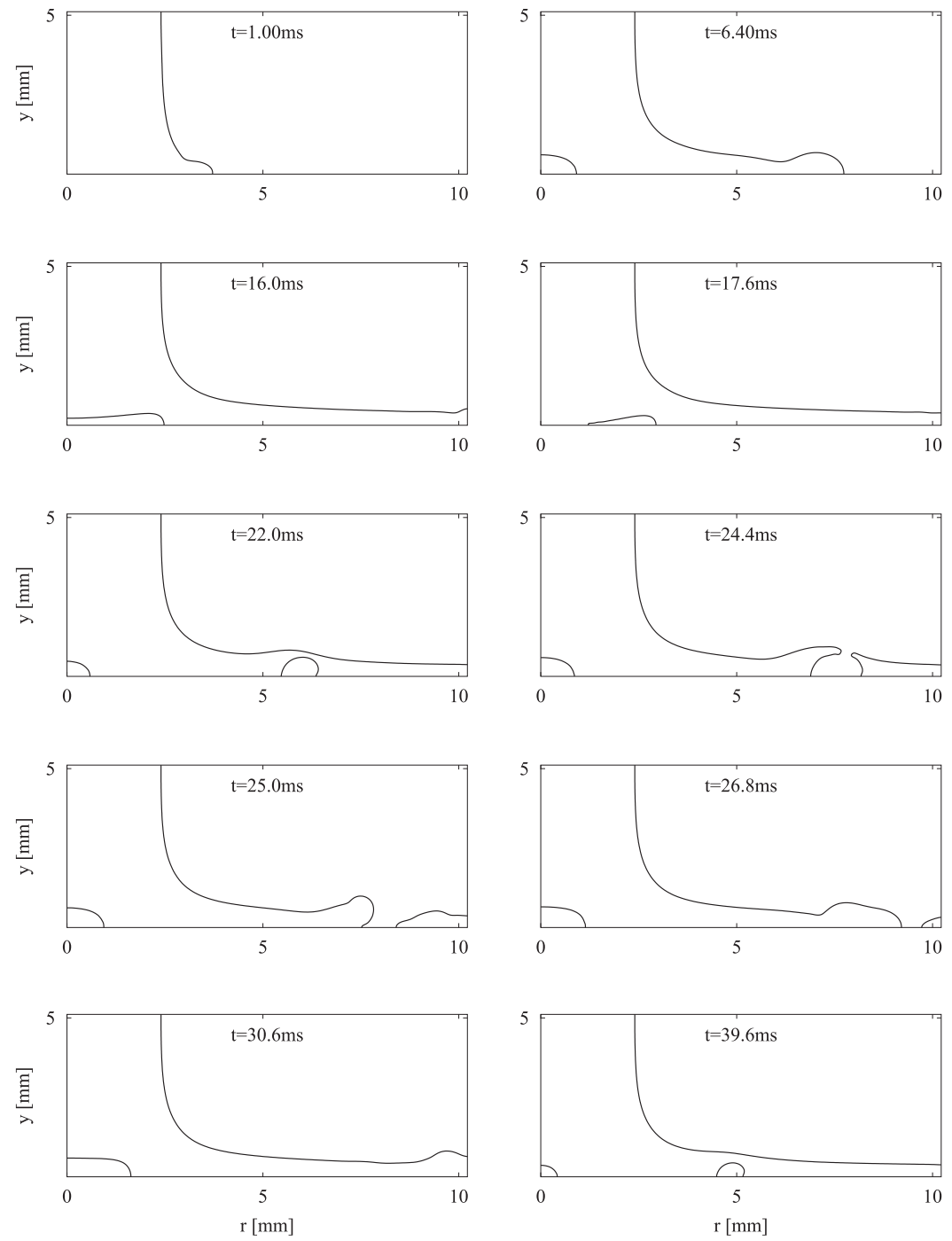

Fig. 2 Evolution of the liquid-air and liquid-vapor interfaces for $V_{j}=1 \mathrm{~m} / \mathrm{s} \Delta T_{w}=$ $200^{\circ} \mathrm{C}$ and $\Delta T_{\text {sub }}=1{ }^{\circ} \mathrm{C}$

\section{Results and Discussion}

In carrying out the calculations, we use the water and air properties at $1 \mathrm{~atm}$. The computational domain is chosen as a cylindrical region which has a radius of $R$ and a height of $H$. We use a nozzle diameter of $D_{j}=4.8 \mathrm{~mm}$ and a domain size of $R=10.24 \mathrm{~mm}$ and $H=5.12 \mathrm{~mm}$. We parametrically vary the jet velocity $V_{j}$, the wall superheat $\Delta T_{w}=T_{w}-T_{s a t}$, and the jet subcooling $\Delta T_{\text {sub }}=T_{\text {sat }}-T_{j}$. As a preliminary attempt to simulate the bubble growth in jet impingement, we consider a single bubble nucleation site, which is located at the center of plate, $(r, y)=(0,0)$. A bubble with a diameter of $0.2 \mathrm{~mm}$ is placed on the site when it is occupied by a superheated liquid $\left(T>T_{\text {sat }}\right)$ during the waiting period $\tau_{w}$ for bubble generation. We use $\tau_{w}=1 \mathrm{~ms}$. During the computations, we use a uniform grid spacing of $h=0.04 \mathrm{~mm}$. When grid resolutions are tested with $h=0.04 \mathrm{~mm}$ and $h=0.02 \mathrm{~mm}$, the area and time averaged wall heat fluxes for both meshes differ by less than $6 \%$.

\subsection{Interfacial motion and the associated flow and heat transfer}

Figure 2 shows the evolution of the liquid-gas interfaces including bubble motion for $V_{j}=1 \mathrm{~m} / \mathrm{s}, \Delta T_{w}=200^{\circ} \mathrm{C}$ and $\Delta T_{s u b}=1{ }^{\circ} \mathrm{C}$. As a bubble placed at $t=\tau_{w}$ grows, it elongates into the radial direction at $t=16.0 \mathrm{~ms}$ because of the impinging jet, and then departs from 

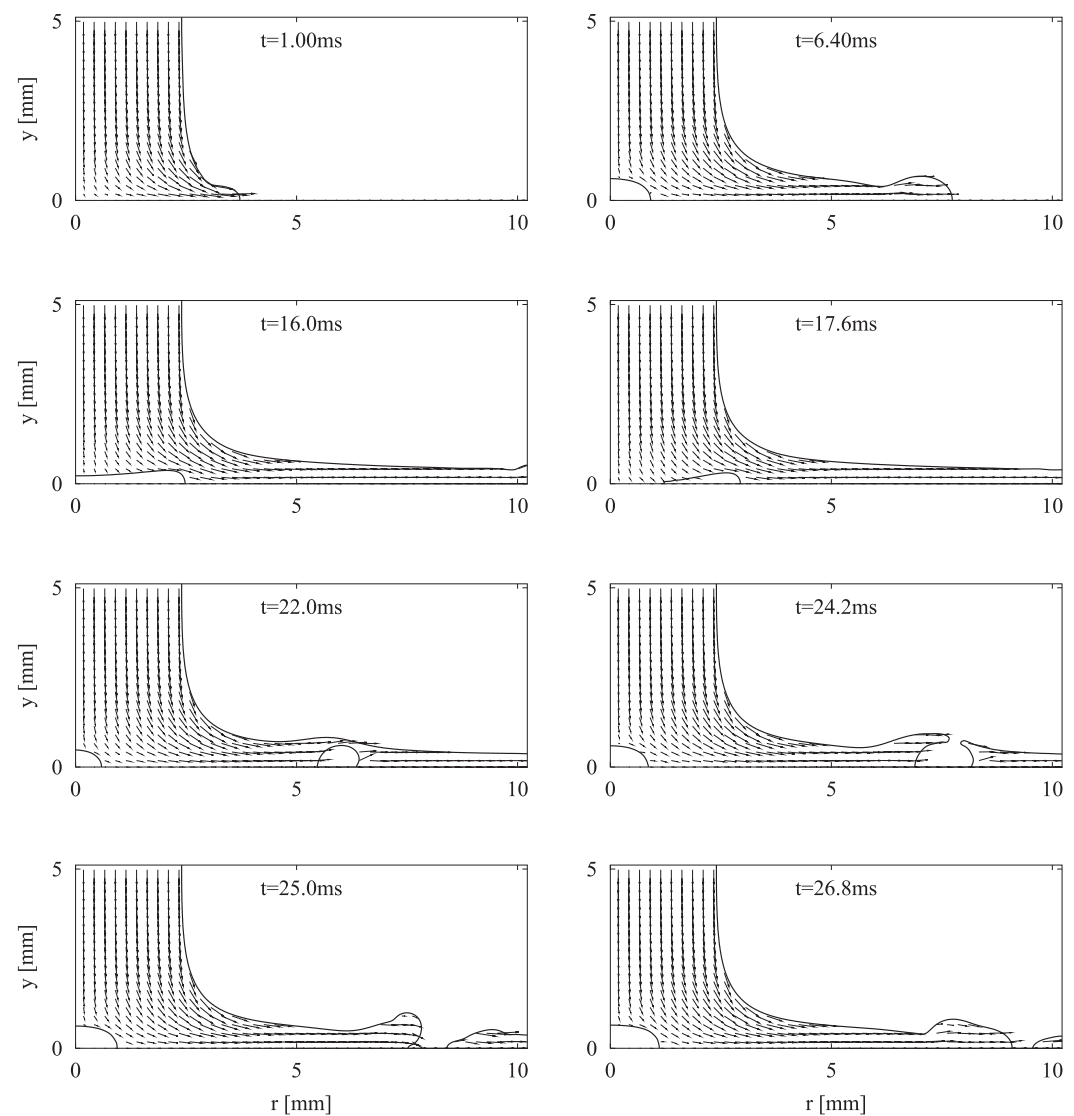

Fig. 3 Liquid flow field for $V_{j}=1 \mathrm{~m} / \mathrm{s}, \Delta T_{w}=200^{\circ} \mathrm{C}$ and $\Delta T_{s u b}=1{ }^{\circ} \mathrm{C}$

the jet impact point. The departed bubble moves radially outwards with time. Its growth rate increases as the bubble comes out from the stagnation region near the jet impact and the liquid temperature rises up. For $22.0 \mathrm{~ms} \leq t \leq 24.4 \mathrm{~ms}$, the bubble meets the liquid-air interface and is sucked into the air bulk. Thereafter a new bubble, which is placed on the bubble nucleation site after the end of the waiting period, grows in the liquid jet. The bubble growth pattern, including the departure from the jet impact point and merger with the air bulk, repeats all over again.

The liquid flow field associated with the bubble growth and the liquid-air interfacial motion is plotted in Figure 3. In the stagnation region, the liquid velocity near the wall is smaller than the impact jet velocity $V_{j}$. During the initial period of $t<6.4$, the liquid velocity field is little affected by the bubble near $r=0$. The interaction between the bubble motion and the liquid flow velocity becomes pronounced as the bubble grows and comes off the jet impact region.

Figure 4 shows the temperature field in the liquid and air regions. The bubble temperature is assumed to remain at the saturation temperature, as mentioned earlier. The isotherms are closely packed near the wall occupied by the impinging liquid jet when compared with the air region. This reflects the high heat flux in the liquid region. The bubble height is observed to be much larger than the boundary thickness of superheated liquid $\left(T>T_{\text {sat }}\right)$. This means that evaporation occurs on the lower portion of bubble surface while condensation occurs on the upper portion. The bubble grows or shrinks depending on the temperature distribution near the bubble. As the bubble moves outwards ( $17 m s \leq t \leq 22 \mathrm{~ms}$ ), the thermal boundary is thicker near the front of bubble. This results in higher heat transfer into the bubble and the bubble growth is sped up.

The wall heat flux averaged over the plate is plotted in Figure 5. The averaged wall heat 

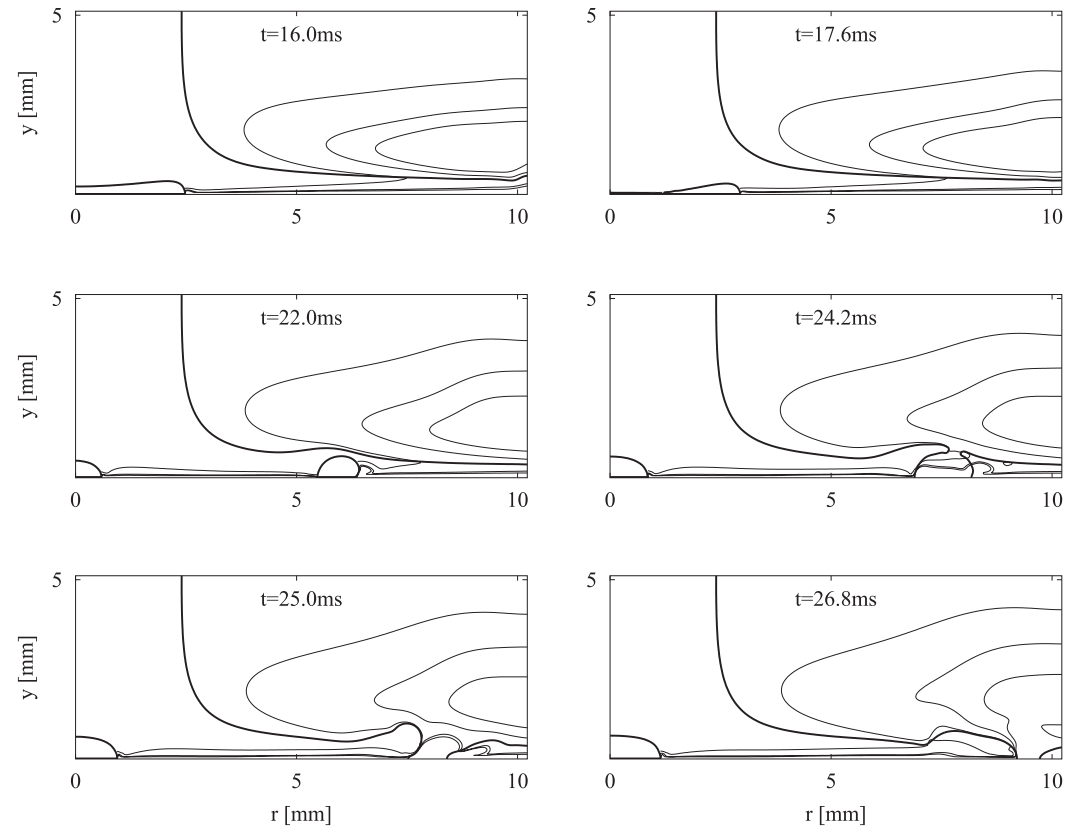

Fig. 4 Temperature field for $V_{j}=1 \mathrm{~m} / \mathrm{s}, \Delta T_{w}=200^{\circ} \mathrm{C}$ and $\Delta T_{\text {sub }}=1{ }^{\circ} \mathrm{C}$. The isotherms represent $100^{\circ} \mathrm{C}, 105^{\circ} \mathrm{C}$, and $110^{\circ} \mathrm{C}$.

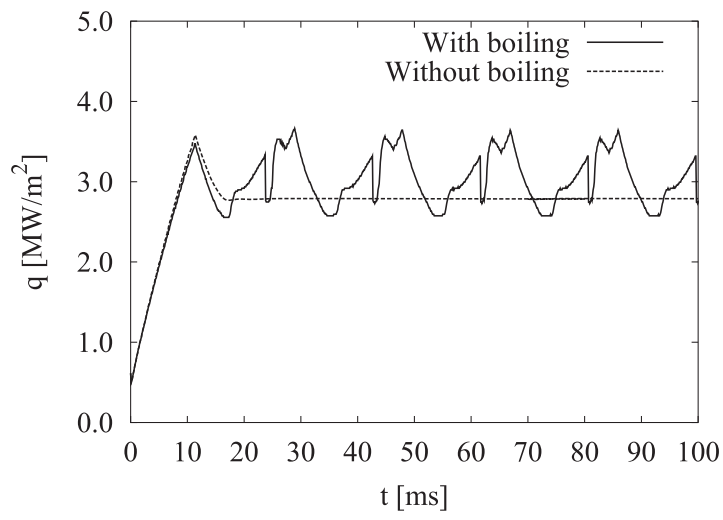

Fig. 5 Area-averaged wall heat flux for $V_{j}=1 \mathrm{~m} / \mathrm{s}, \Delta T_{w}=200^{\circ} \mathrm{C}$ and $\Delta T_{s u b}=1{ }^{\circ} \mathrm{C}$

flux is calculated as $-\left.\int_{0}^{R} 2 r\left(\lambda+\rho c_{p} v_{t} / P r_{T}\right) \frac{\partial T}{\partial y}\right|_{y=0} d r / R^{2}$. The heat flux for jet impingement including boiling or bubble growth shows periodic oscillation whereas the heat flux without boiling reaches a steady state. Initially, the wall heat flux increases linearly as the liquid film expands along the plate. When the liquid film expands beyond the outlet boundary of the computational domain and the liquid is heated up, the wall heat flux decreases, as seen in $11 \mathrm{~ms}<t<17 \mathrm{~ms}$. During this period, as the bubble formed at $r=0$ grows into the radial direction and the low heat transfer region occupied by the vapor increases, the associated wall heat flux tends to be lower than that without boiling. While the bubble departs and slides outwards, as seen in $17 m s<t<24 m s$, the wall heat flux increases. This indicates the sliding bubble enhances the heat transfer significantly. At $t=24 \mathrm{~ms}$, when the bubble is merged with air bulk, the heat flux decreases rapidly. The wall heat flux is recovered as the area vacated by the sucking bubble is re-wetted by the liquid. Thereafter, the bubble growth and associated heat flux variation patterns repeat all over again with a new bubble. The area and time averaged heat flux is $3.05 \mathrm{MW} / \mathrm{m}^{2}$ for the case with boiling, which is $9.7 \%$ larger than the case without boiling. 

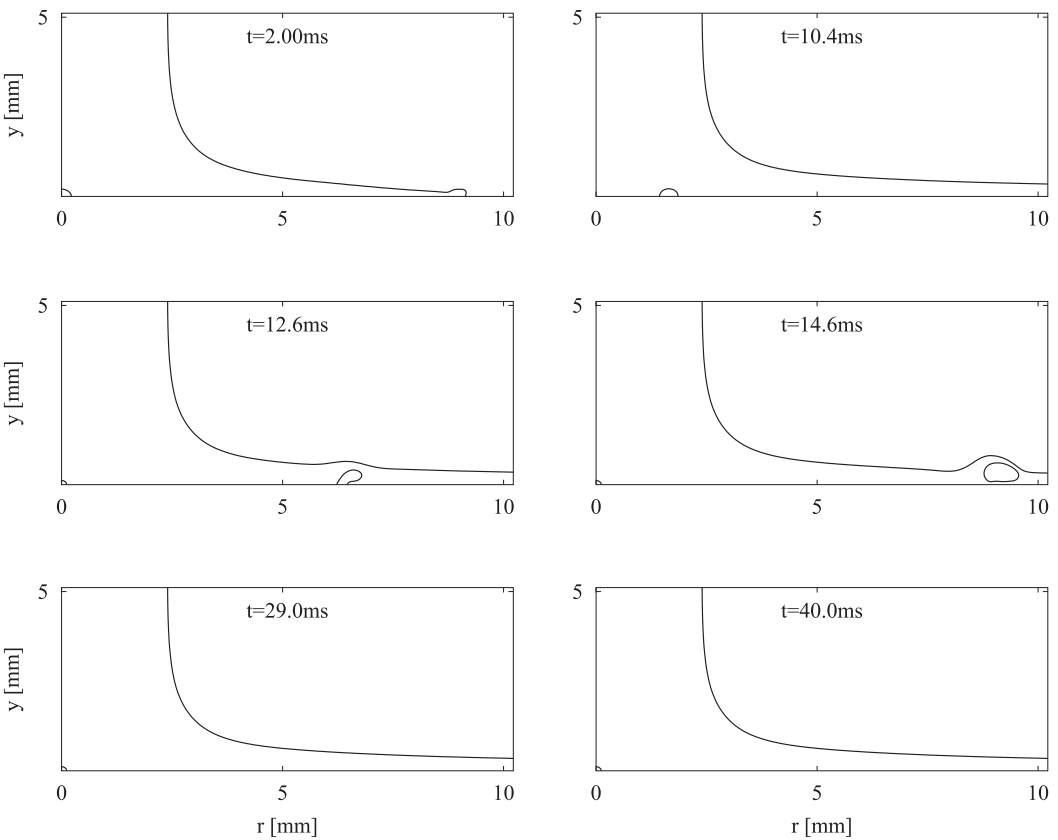

Fig. 6 Evolution of the liquid-air and liquid-vapor interfaces for $V_{j}=3 \mathrm{~m} / \mathrm{s}, \Delta T_{w}=$ $200^{\circ} \mathrm{C}$ and $\Delta T_{s u b}=1^{\circ} \mathrm{C}$

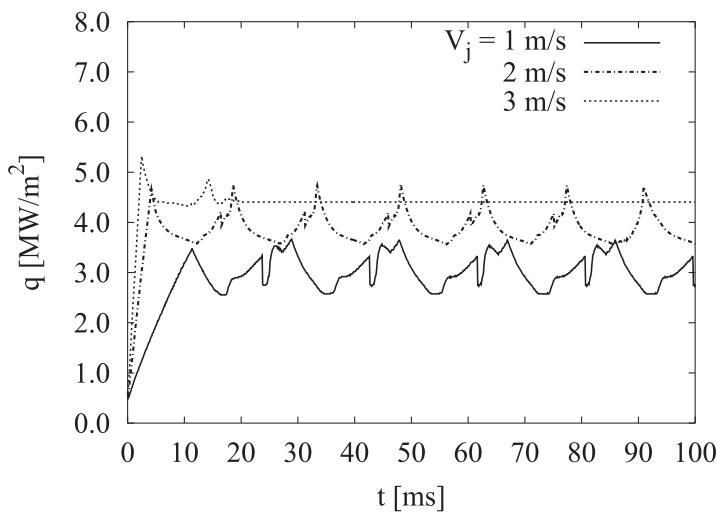

Fig. 7 Effect of jet velocity on the wall heat flux for $\Delta T_{w}=200^{\circ} \mathrm{C}$ and $\Delta T_{\text {sub }}=1^{\circ} \mathrm{C}$

\subsection{Effect of jet velocity}

Figure 6 presents the interfacial motion when the jet velocity is increased to $V_{j}=3 \mathrm{~m} / \mathrm{s}$. The pattern of bubble growth, departure and merger with air during the first cycle $(0 \leq t \leq$ 14.6) is similar to the case of $V_{j}=1 \mathrm{~m} / \mathrm{s}$, except that the departed bubble size is decreased. However, the second bubble for the next cycle does not grow as the effect of condensation is pronounced with the increase in jet velocity.

The effect of jet velocity on the area-averaged wall heat flux is depicted in Figure 7. As the jet velocity increases, the heat flux is observed to increase. The periodic oscillation of heat flux caused by the cyclic pattern of bubble growth does not appear in the case of $V_{j}=3 \mathrm{~m} / \mathrm{s}$. The area and time averaged heat fluxes for $V_{j}=1 \mathrm{~m} / \mathrm{s}, 2 \mathrm{~m} / \mathrm{s}$ and $3 \mathrm{~m} / \mathrm{s}$ are $3.05 \mathrm{MW} / \mathrm{m}^{2}$, $3.90 \mathrm{MW} / \mathrm{m}^{2}$ and $4.41 \mathrm{MW} / \mathrm{m}^{2}$, respectively. The heat flux varies as $V_{j}^{0.33}$.

\subsection{Effect of wall temperature (superheat)}

Figure 8 shows the bubble growth pattern when $\Delta T_{w}$ is decreased to $100^{\circ} \mathrm{C}$. A bubble 

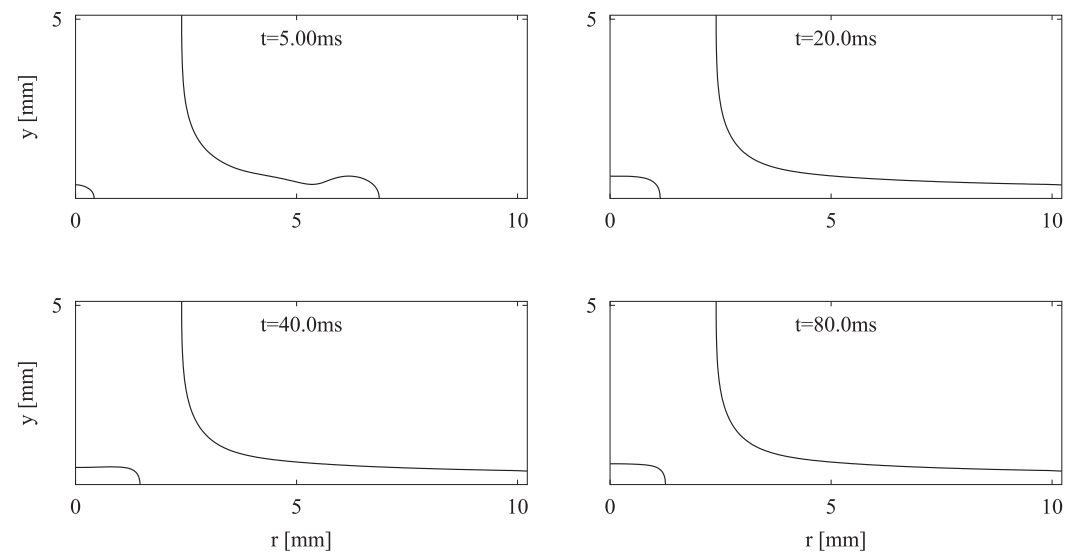

Fig. 8 Evolution of the liquid-air and liquid-vapor interfaces for $\Delta T_{w}=100^{\circ} \mathrm{C}$, $V_{j}=1 \mathrm{~m} / \mathrm{s}$ and $\Delta T_{s u b}=1{ }^{\circ} \mathrm{C}$
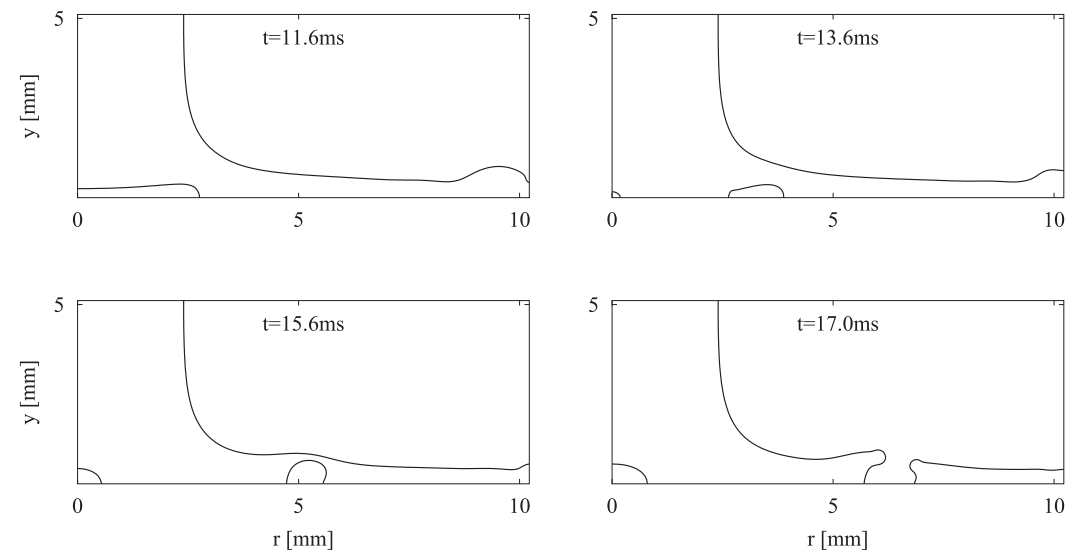

Fig. 9 Evolution of the liquid-air and liquid-vapor interfaces for $\Delta T_{w}=300^{\circ} \mathrm{C}$, $V_{j}=1 \mathrm{~m} / \mathrm{s}$ and $\Delta T_{\text {sub }}=1^{\circ} \mathrm{C}$

placed at the nucleation site, $(r, y)=(0,0)$, grows initially, but its growth does not continue as the evaporation rate from the lower portion of bubble surface is balanced with the condensation rate from the upper portion. The bubble remains near the jet impact region while its shape and volume are oscillating slightly. Figure 9 presents the interfacial motion for a higher wall superheat of $\Delta T_{w}=300^{\circ} \mathrm{C}$. The cyclic pattern of bubble growth, departure and merger with air is similar to the case of $\Delta T_{w}=200^{\circ} \mathrm{C}$, except that the departed bubble becomes larger and the cycle frequency increases.

The effect of wall superheat on the area-averaged wall heat flux is plotted in Figure 10. The area and time averaged heat fluxes for $\Delta T_{w}=100^{\circ} \mathrm{C}, 200^{\circ} \mathrm{C}$ and $300^{\circ} \mathrm{C}$ are $1.38 \mathrm{MW} / \mathrm{m}^{2}$, $3.05 \mathrm{MW} / \mathrm{m}^{2}$ and $4.62 \mathrm{MW} / \mathrm{m}^{2}$, respectively. The wall heat flux is observed to increase almost linearly with $\Delta T_{w}$. This means that the boiling heat transfer coefficient does not change with wall superheat as long as the nucleation site density and waiting time remain fixed.

\subsection{Effect of jet temperature (subcooling)}

Figure 11 shows the evolution of liquid-gas interfaces for a higher jet subcooling of $\Delta T_{\text {sub }}=5^{\circ} \mathrm{C}$. The observed stationary bubble motion with slight oscillation is similar to the case for a lower wall superheat of $\Delta T_{w}=200^{\circ} \mathrm{C}$. The effect of jet subcooling on the areaaveraged wall heat flux is depicted in Figure 12. The heat flux varies periodically with the cyclic behavior of bubble growth while its oscillation amplitude depends on the jet subcool- 


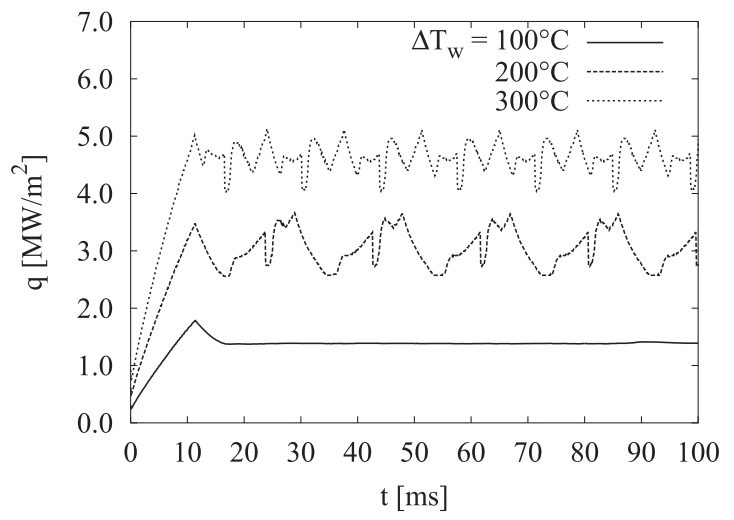

Fig. 10 Effect of wall superheat on the wall heat flux for $V_{j}=1$ and $\Delta T_{\text {sub }}=1^{\circ} \mathrm{C}$
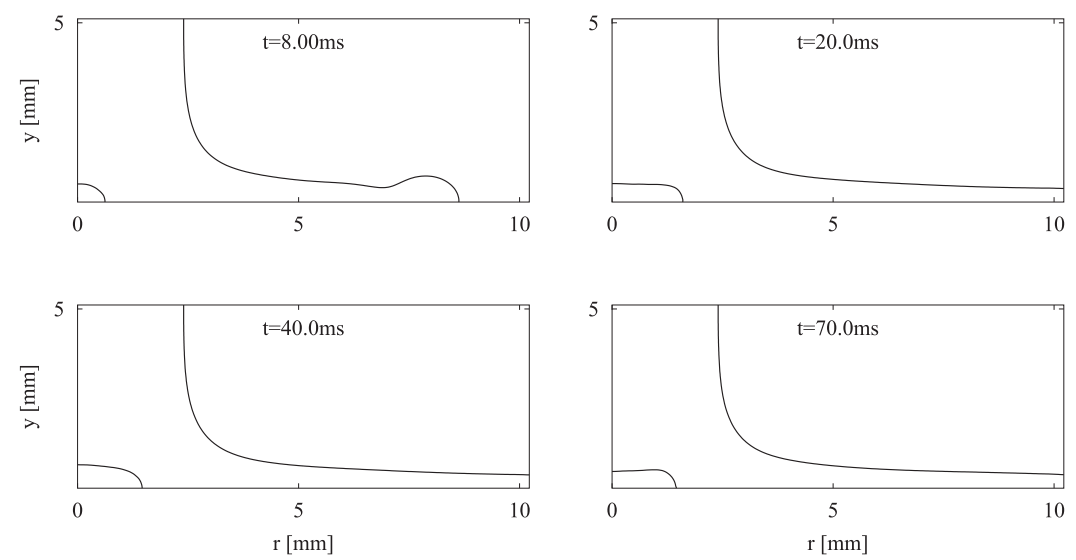

Fig. 11 Evolution of the liquid-air and liquid-vapor interfaces for $\Delta T_{\text {sub }}=5^{\circ} \mathrm{C}$, $V_{j}=1 \mathrm{~m} / \mathrm{s}$ and $\Delta T_{w}=200^{\circ} \mathrm{C}$

ing. The area and time averaged heat fluxes for $\Delta T_{\text {sub }}=1{ }^{\circ} \mathrm{C}, 3^{\circ} \mathrm{C}$ and $5^{\circ} \mathrm{C}$ are $3.05 \mathrm{MW} / \mathrm{m}^{2}$, $2.97 \mathrm{MW} / \mathrm{m}^{2}$ and $2.82 \mathrm{MW} / \mathrm{m}^{2}$, respectively. This indicates that the heat flux is nearly independent of the jet subcooling in the range of $\Delta T_{\text {sub }} \leq 5^{\circ} \mathrm{C}$.

\section{Conclusion}

A numerical approach was developed for complete numerical simulation of boiling in free-surface liquid jet impingement on a hot plate. The level-set method for tracking the liquid-gas interfaces was extended to include the effect of phase change at the liquid-vapor interface and a non-equilibrium $k-\varepsilon$ turbulence model. The numerical method was applied to computation of bubble growth in a water jet assuming a single nucleation site located at the center of plate and a constant waiting period for bubble generation.

The typical numerical results demonstrated a cyclic pattern of bubble growth, including the departure from the jet impact region and merger with the liquid-air interface. The wall heat flux varies periodically during the bubble growth and its magnitude is larger than the case without including the boiling effect. The bubble growth pattern coupled to boiling heat transfer strongly depends on the effects of jet velocity and wall superheat. The wall heat heat flux is observed to increase almost linearly with the wall superheat whereas it is nearly independent of the jet subcooling.

\section{Acknowledgements}

This work was supported by the National Research Foundation of Korea (NRF) grant No. 20090083510 funded by the Korean government (MEST) through Multi-phenomena CFD Engineering Research Center. 


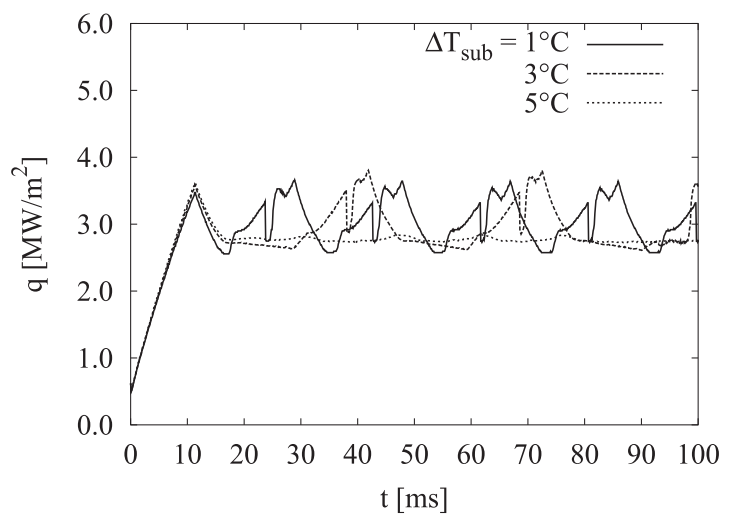

Fig. 12 Effect of jet subcooling on the wall heat flux for $V_{j}=1 \mathrm{~m} / \mathrm{s}$ and $\Delta T_{w}=200^{\circ} \mathrm{C}$

\section{References}

( 1 ) Wolf D.H., Incropera F.P., Viskanta R., Jet impingement boiling, Adv. Heat Transfer 23, pp.1-132, 1993

( 2 ) Hall D.E., Incropera F.P., Viskanta R., Jet impingement boiling from a circular freesurface jet during quenching: Part 1-single-phase jet, J. Heat Transfer 123, pp. 901-910, 2001

( 3 ) Karwa N., Gambaryan-Roisman T., Stephan P., Tropera C., Experimental investigation of circular free-surface jet impingement quenching: Transient hydrodynamics and heat transfer, Experimental Thermal and Fluid Science 35, pp. 1435-1443, 2011

( 4 ) Fujimoto H., Hatta N., Viskanta, R., Numerical simulation of convective heat transfer to a radial free surface jet impinging on a hot solid, Heat Mass Transfer 35, pp. 266-272, 1999

( 5 ) Tong A.Y., A numerical study on the hydrodynamics and heat transfer of a circular liquid jet impinging onto a substrate, Numerical Heat Transfer Part A 44, pp. 1-19, 2003

( 6 ) Gradeck M., Kouachi A., Dani A., Amoult D., Borean J., Experimental and numerical study of the hydraulic jump of an impinging jet on a moving Surface, Exp. Therm. Fluid Sci. 30, pp. 193-201, 2006

( 7 ) Son S., Son G., Park I, Lee P., Numerical study of flow and cooling characteristics of impinging liquid jets on a moving plate, Proc. IHTC14, Washington DC, USA, 2009

( 8 ) Mikilewicz J., Mikilewicz D., Barnik D., Modeling of nucleate boiling heat transfer in a film formed by an impingement jet, Heat Transfer Res. 38, pp. 313-323, 2007

( 9 ) Omar A.M.T., Hamed M.S., Shoukri M., Modeling of nucleate boiling heat transfer under an impinging free jet, Int. J. Heat and Mass Transfer 52, pp. 5557-5566, 2009

(10) Naramuch S., Troshko A., Bharathan D., Hassani V., Numerical simulations of nucleate boiling in impinging jets: Application in power electronics cooling, Int. J. Heat and Mass Transfer 51, pp. 1-12, 2008

(11) Lee R.C., Nydahl E., Numerical calculation of bubble growth in nucelate boiling from incetption through departure, J. Heat Transfer 111, pp. 474-479, 1989

(12) Welch S.W.J., Direct simulation of vapor bubble growth, Int. J. Heat and Mass Transfer 41, pp. 1655-1666, 1998

(13) Shin S., Abdel-Khalik S.I., Juric D., Direct three-dimensional numerical simulation of nucleate boiling using the level contour reconstruction method, Int. J. Multiphase Flow 31, pp. 1231-1242, 2005.

(14) Kunkelmann C., Stephan P., CFD simulation of boiling flows using the volume-of-fluid method within OpenFOAM, Numer. Heat Transfer A 56, pp. 631-646, 2009

(15) Son G., Dhir V.K., Ramanujapu N., Dynamics and heat transfer associated with a single bubble during nucleate boiling on a horizontal surface, J. Heat Transfer 121, pp. 623631,1999 
(16) Son G., Dhir V.K., Numerical simulation of nucleate boiling on a horizontal surface at high heat fluxes, Int. J. Heat and Mass Transfer 51, pp. 2566-2582, 2008.

(17) Shyy W., Thakur S.S., Quyang H., Liu J., Blosch, Computational techniques for complex transport, Cambridge, New York, 1997

(18) Suh Y., Son G., A level-set method for simulation of a thermal inkjet process, Numerical Heat Transfer Part B 54, pp. 138-156, 2008 